\title{
Honorary Fellows
}

Dr Najeeb Al-Shorbaji

Switzerland

Prof. Ab Bakker

The Netherlands

Dr. Marion J. Ball

USA

Dieter Bergemann

Germany

Lincoln de Assis Moura Jr

Brazil

Profl. Antoine Geissbuhler

Switzerland

Prof. Malcolm Forsythe

United Kingdom

Prof. W. Ed Hammond

USA

Prof. Reinhold Haux

Germany

Prof. Casimir Kulikowski

USA

Prof. Donald A. B. Lindberg

USA

Dr. Nancy M. Lorenzi

USA
Prof. K.C. Lun

Singapore

Prof. Alexa McCray

USA

Dr. Ramin Moghaddam

Iran

Dr. Peter Murray

United Kingdom

Prof. Hans E. Peterson

Sweden

Prof. Otto Rienhoff

Germany

Dr. Jan Roukens

Belgium

Dr. David Shires

USA

Prof. Edward H. Shortliffe

USA

Prof. Jan H. van Bemmel

The Netherlands

Prof. Valerio Yacubsohn

Argentina

The following IMIA Honorary Fellows have passed away, but we list them in continuing recognition of their contribution to IMIA:

William C. Abbott, United Kingdom

Prof. Branko Cesnik, Australia

Dr. Morris F. Collen, USA

François Grémy, France

Steven A. Huesing, Canada
Dr. Sedick Isaacs, South Africa

Prof. Shigekoto Kaihara, Japan

Mr. Ian H. Symonds, New Zealand

Dr. Jos Willems, Belgium 